\title{
BOUNDARY-VALUE PROBLEMS FOR LINEAR EQUATIONS WITH A GENERALIZED INVERTIBLE OPERATOR IN A BANACH SPACE WITH BASIS
}

\author{
V.F. Zhuravlev
}

UDC 517.983

\begin{abstract}
We consider linear boundary-value problems for operator equations with generalized invertible operators in Banach spaces that have bases. Using the technique of generalized inverse operators applied to generalized invertible operators in Banach spaces, we establish conditions for the solvability of linear boundary-value problems for these operator equations and obtain formulas for the representation of their solutions. We consider special cases of these boundary-value problems, namely, so-called $n$ - and $d$-normally solvable boundary-value problems as well as normally solvable problems for Noetherian operator equations.
\end{abstract}

\section{Statement of the Problem}

Let $\mathbf{l}_{\infty}\left(\mathcal{I}, \mathbf{B}_{1}\right)$ be the Banach space of bounded vector functions $z(t)$ defined on a finite interval $\mathcal{I}$ and taking values in a certain Banach space $\mathbf{B}_{1}, z(\cdot): \mathcal{I} \rightarrow \mathbf{B}_{1}$, with the norm

$$
\|f(t)\|=\sup _{t \in \mathcal{I}}\|f(t)\|_{\mathbf{B}_{1}}
$$

and let $\mathbf{l}_{\infty}\left(\mathcal{I}, \mathbf{B}_{2}\right)$ be the Banach space of bounded vector functions $\varphi(t)$ defined on the same interval $\mathcal{I}$ and taking values in a certain Banach space $\mathbf{l}_{\infty}\left(\mathcal{I}, \mathbf{B}_{2}\right)$ with the norm

$$
\|f(t)\|\left\|=\sup _{t \in \mathcal{I}}\right\| f(t) \|_{\mathbf{B}_{2}}
$$

Assume that $L$ is a linear bounded generalized invertible operator that acts from the Banach space $\mathbf{l}_{\infty}\left(\mathcal{I}, \mathbf{B}_{1}\right)$ into the Banach space $\mathbf{l}_{\infty}\left(\mathcal{I}, \mathbf{B}_{2}\right)$, the spaces $\mathbf{l}_{\infty}\left(\mathcal{I}, \mathbf{B}_{1}\right)$ and $\mathbf{l}_{\infty}\left(\mathcal{I}, \mathbf{B}_{2}\right)$ have bases, and $\ell=\operatorname{col}\left(l_{1}, l_{2}, l_{3}, \ldots\right)$ is a linear bounded vector functional that acts from the Banach space $\mathbf{l}_{\infty}\left(\mathcal{I}, \mathbf{B}_{1}\right)$ into the Banach space $\mathbf{B}$.

It follows from the generalized invertibility of the operator $L[1]$ that $L$ is normally solvable $(R(L)$ is closed), and the null space $N(L)$ and the image $R(L)$ are complementable in the Banach spaces $\mathbf{l}_{\infty}\left(\mathcal{I}, \mathbf{B}_{1}\right)$ and $\mathbf{l}_{\infty}\left(\mathcal{I}, \mathbf{B}_{2}\right)$, respectively. This means [2,3] that there exist linear bounded projectors $\mathcal{P}_{N(L)}: \mathbf{l}_{\infty}\left(\mathcal{I}, \mathbf{B}_{1}\right) \rightarrow$ $N(L)$ and $\mathcal{P}_{Y_{L}}: \mathbf{l}_{\infty}\left(\mathcal{I}, \mathbf{B}_{2}\right) \rightarrow Y_{L}$ that decompose the Banach spaces $\mathbf{l}_{\infty}\left(\mathcal{I}, \mathbf{B}_{1}\right)$ and $\mathbf{l}_{\infty}\left(\mathcal{I}, \mathbf{B}_{2}\right)$ into mutually complementing subspaces, namely,

$$
\mathbf{l}_{\infty}\left(\mathcal{I}, \mathbf{B}_{1}\right)=N(L) \oplus X_{L}, \quad \mathbf{l}_{\infty}\left(\mathcal{I}, \mathbf{B}_{2}\right)=Y_{L} \oplus R(L)
$$

where $Y_{L}$ is a subspace isomorphic to the null space $N\left(L^{*}\right)$ of the operator $L^{*}$ adjoint to the operator $L$.

Zhitomir National Agroecological University, Zhitomir, Ukraine; e-mail: vfz2008@ukr.net.

Translated from Neliniini Kolyvannya, Vol. 13, No. 4, pp. 522-532, October-December, 2010. Original article submitted March 3, 2010. 
In what follows, we denote the set of generalized invertible operators $L: \mathbf{l}_{\infty}\left(\mathcal{I}, \mathbf{B}_{1}\right) \rightarrow \mathbf{l}_{\infty}\left(\mathcal{I}, \mathbf{B}_{2}\right)$ by $G I\left(\mathbf{l}_{\infty}\left(\mathcal{I}, \mathbf{B}_{1}\right), \mathbf{l}_{\infty}\left(\mathcal{I}, \mathbf{B}_{2}\right)\right)$.

Since the Banach spaces $\mathbf{l}_{\infty}\left(\mathcal{I}, \mathbf{B}_{1}\right)$ and $\mathbf{l}_{\infty}\left(\mathcal{I}, \mathbf{B}_{2}\right)$ have bases, the null spaces $N(L)$ and $N\left(L^{*}\right)$ also have bases.

Let

$$
\left\{f_{i}\right\}_{i=1}^{\infty} \subset N(L), \quad f_{i}=\operatorname{col}\left(f_{i}^{(1)}, f_{i}^{(2)}, f_{i}^{(3)}, \ldots\right),
$$

and

$$
\left\{\varphi_{s}(\cdot)\right\}_{s=1}^{\infty} \subset N\left(L^{*}\right), \quad \varphi_{s}(\cdot)=\operatorname{col}\left(\varphi_{s}^{(1)}(\cdot), \varphi_{s}^{(2)}(\cdot), \varphi_{s}^{(3)}(\cdot), \ldots\right),
$$

denote the bases of the null spaces $N(L)$ and $N\left(L^{*}\right)$, respectively. For elements $\left\{f_{i}\right\}_{i=1}^{\infty}$ and functionals $\left\{\varphi_{s}\right\}_{s=1}^{\infty}$, there exist an adjoint biorthogonal [4] total system of functionals $\left\{\gamma_{j}\right\}_{j=1}^{\infty} \subset N^{*}(L) \subset \mathbf{l}_{\infty}\left(\mathcal{I}, \mathbf{B}_{1}\right)^{*}$, $\gamma_{j}(\cdot)=\operatorname{col}\left(\gamma_{j}^{(1)}(\cdot), \gamma_{j}^{(2)}(\cdot), \gamma_{j}^{(3)}(\cdot), \ldots\right)$, and an adjoint biorthogonal compete system of elements $\left\{\psi_{k}\right\}_{k=1}^{\infty} \subset$ $\mathbf{l}_{\infty}\left(\mathcal{I}, \mathbf{B}_{2}\right), \quad \psi_{k}=\operatorname{col}\left(\psi_{k}^{(1)}, \quad \psi_{k}^{(2)}, \psi_{k}^{(3)}, \ldots\right)$. According to the Hahn-Banach theorem, each of the functionals $\left\{\gamma_{j}\right\}_{j=1}^{\infty}$ defined on the subspace $N(L) \subset \mathbf{l}_{\infty}\left(\mathcal{I}, \mathbf{B}_{1}\right)$ can be extended to the entire space $\mathbf{l}_{\infty}\left(\mathcal{I}, \mathbf{B}_{1}\right)$ with preservation of norm, and each of the functionals $\left\{\varphi_{s}\right\}_{s=1}^{\infty}$ can be extended to the entire space $\mathbf{l}_{\infty}\left(\mathcal{I}, \mathbf{B}_{2}\right)$.

Let $X=\left(f_{1}, f_{2}, \ldots, f_{i}, \ldots\right), \Gamma(\cdot)=\left(\gamma_{1}(\cdot), \gamma_{2}(\cdot), \ldots, \gamma_{j}(\cdot), \ldots\right)^{T}, \Phi(\cdot)=\left(\varphi_{1}(\cdot), \varphi_{2}(\cdot), \ldots, \varphi_{s}(\cdot), \ldots\right)^{T}$, and $\Psi=\left(\psi_{1}, \psi_{2}, \ldots, \psi_{k}, \ldots\right)$ be $(\infty, \infty)$-dimensional matrix operators; here, $\Gamma(X)=E_{\infty}, \Phi(\Psi)=E_{\infty}$, and $E_{\infty}$ is the identity matrix.

Then the projectors $\mathcal{P}_{N(L)}: \mathbf{l}_{\infty}\left(\mathcal{I}, \mathbf{B}_{1}\right) \rightarrow N(L)$ and $\mathcal{P}_{Y_{L}}: \mathbf{l}_{\infty}\left(\mathcal{I}, \mathbf{B}_{2}\right) \rightarrow Y_{L}$ can be represented as follows [5, pp. 168, 172]:

$$
\begin{aligned}
& \left(\mathcal{P}_{N(L)} z\right)(t)=X(t)(\Gamma z)(\cdot) \quad \forall z \in \mathbf{l}_{\infty}\left(\mathcal{I}, \mathbf{B}_{1}\right), \\
& \left(\mathcal{P}_{Y_{L}} y\right)(t)=\Psi(t)(\Phi y)(\cdot) \quad \forall y \in \mathbf{l}_{\infty}\left(\mathcal{I}, \mathbf{B}_{2}\right) .
\end{aligned}
$$

We consider the problem of conditions for the solvability of the operator equation

$$
(L i z)(t)=\varphi(t)
$$

and the representation of its solutions that satisfy the conditions

$$
(\ell z)(\cdot)=\alpha,
$$

where $\alpha \in \mathbf{B}$.

Definition 1. The system of linear operator equations (1), (2) is called the linear inhomogeneous boundaryvalue problem for Eq. (1), and the equations

$$
(\ell z)(\cdot)=\operatorname{col}\left(\left(l_{1} z\right)(\cdot),\left(l_{2} z\right)(\cdot),\left(l_{3} z\right)(\cdot), \ldots,\left(l_{i} z\right)(\cdot), \ldots\right)=\operatorname{col}\left(\alpha_{1}, \alpha_{2}, \alpha_{3}, \ldots, \alpha_{i}, \ldots\right)
$$

are called the boundary conditions of this problem. 


\section{Intermediate Result}

Consider the problem of conditions for the solvability of the operator equation

$$
(L z)(t)=\varphi(t)
$$

and the general form of its solutions that satisfy the initial condition

$$
z\left(t_{0}\right)=z_{0},
$$

where $t_{0} \in \mathcal{I}$ and $z_{0} \in \mathbf{B}_{1}$.

Definition 2. Problem (4), (5) is called the Cauchy problem for the inhomogeneous operator equation (4).

It is known [6] that the operator equation (4) is solvable for those and only those $\varphi(t) \in \mathbf{l}_{\infty}\left(\mathcal{I}, \mathbf{B}_{2}\right)$ that satisfy the condition

$$
\left(\mathcal{P}_{Y_{L}} \varphi\right)(t)=\Psi(t)(\Phi \varphi)(\cdot)=0
$$

Under condition (6), the general solution of Eq. (4) can be represented in the form [6]

$$
z(t)=\left(\mathcal{P}_{N(L)} \hat{z}\right)(t)+\left(L^{-} \varphi\right)(t)
$$

where $\hat{z}(t)$ is an arbitrary element of the space $\mathbf{l}_{\infty}\left(\mathcal{I}, \mathbf{B}_{1}\right)$ and $L^{-}$is the bounded generalized inverse of $L$, the construction of which is described in [5-8] for operators $L$ of various types.

Using representation (1) for the projector $\mathcal{P}_{N(L)}$, for every $\tilde{z}(t) \in N(L)$ we get

$$
\left(\mathcal{P}_{N(L)} \hat{z}\right)(t)=X(t)(\Gamma \hat{z})(\cdot)=X(t) \hat{z}_{0},
$$

where $\hat{z}_{0}=\operatorname{col}\left(\left(\gamma_{1} \hat{z}\right)(\cdot),\left(\gamma_{2} \hat{z}\right)(\cdot), \ldots,\left(\gamma_{j} \hat{z}\right)(\cdot), \ldots\right)$ is an arbitrary column vector. It was shown in [9, p. 129] that, with a properly chosen norm, the linear space whose elements are numerical sequences $\hat{z}_{0}$ for which equality (8) holds is a Banach space. In what follows, we denote this Banach space by $\mathbf{B}_{\mu}$, where $\mu$ is the (finite or infinite) dimension of the kernel of the operator $L$.

Substituting (8) into (7), we obtain the following general solution of the operator equation (4):

$$
z(t)=X(t) \hat{z}_{0}+\left(L^{-} \varphi\right)(t)
$$

It follows from (8) that every element $z(t)$ of the null space $N(L)$ can be represented in the form

$$
z(t)=X(t) \hat{z}_{0}
$$

This relation holds for any $t \in \mathcal{I}$, including $t=t_{0}$. Therefore, for any $z_{0}=z\left(t_{0}\right)$, there exists an element $\hat{z}_{0} \in \mathbf{B}_{\mu}$ such that

$$
z_{0}=X\left(t_{0}\right) \hat{z}_{0},
$$

where $X\left(t_{0}\right): \mathbf{B}_{\mu} \rightarrow \mathbf{B}_{1}$ is a linear bounded operator. 
The existence of the element $\hat{z}_{0}$ for any $z_{0}=z\left(t_{0}\right)$ implies that the matrix equation

$$
X\left(t_{0}\right) \hat{z}_{0}=z_{0}
$$

is everywhere solvable, i.e., (a) $N\left(X^{*}\left(t_{0}\right)\right)=\{0\}$, and, hence, the projector to the subspace $Y_{X\left(t_{0}\right)} \subseteq \mathbf{B}_{1}$ is equal to zero, $\mathcal{P}_{Y_{X\left(t_{0}\right)}} \equiv 0 \forall t_{0} \in \mathcal{I}$, and (b) there exists the right inverse operator $X_{r}^{-1}\left(t_{0}\right)$. Thus, the matrix operator $X\left(t_{0}\right)$ is $d$-normal $(d=0)$ if $\operatorname{dim} \operatorname{ker} X\left(t_{0}\right)=\infty$ or Noetherian if $\operatorname{dim} \operatorname{ker} X\left(t_{0}\right)<\infty$ and $X\left(t_{0}\right)$ is a generalized invertible operator, i.e., $\left.X\left(t_{0}\right) \in G I\left(\mathbf{B}_{\mu}, \mathbf{B}_{1}\right)\right)$.

Thus, the matrix operator equation

$$
X\left(t_{0}\right) \hat{z}_{0}=z_{0}-\left(L^{-} \varphi\right)\left(t_{0}\right),
$$

which is obtained from Eq. (9) for $t=t_{0}$, is everywhere solvable, and its general solution has the form [5, p. 175]

$$
\hat{z}_{0}=\mathcal{P}_{N\left(X\left(t_{0}\right)\right)} z_{\mu}+X_{r}^{-1}\left(t_{0}\right)\left[z_{0}-\left(L^{-} \varphi\right)\left(t_{0}\right)\right]
$$

where $\mathcal{P}_{N\left(X\left(t_{0}\right)\right)}$ is the projector of the Banach space $\mathbf{B}_{\mu}$ to the null space $N\left(X\left(t_{0}\right)\right)$ of the operator $X\left(t_{0}\right)$, $z_{\mu}$ is an arbitrary element of the Banach space of sequences $\mathbf{B}_{\mu}$, and $X_{r}^{-1}\left(t_{0}\right)$ is the right inverse of the matrix operator $X\left(t_{0}\right)$.

Substituting (11) into (9), we obtain the general solution of the Cauchy problem (4), (5):

$$
\begin{aligned}
z(t) & =X(t)\left\{\mathcal{P}_{N\left(X\left(t_{0}\right)\right)} z_{\mu}+X_{r}^{-1}\left(t_{0}\right)\left[z_{0}-\left(L^{-} \varphi\right)\left(t_{0}\right)\right]\right\}+\left(L^{-} \varphi\right)(t) \\
& =X_{0}(t) z_{\mu}+G_{0}(t)+X(t) X_{r}^{-1}\left(t_{0}\right) z_{0},
\end{aligned}
$$

where $X_{0}(t)=X(t) \mathcal{P}_{N\left(X\left(t_{0}\right)\right)}$ is the resolving operator [3] of the homogeneous problem $\left(\varphi(t)=0, z_{0}=0\right)$, $z_{\mu}$ is an arbitrary vector from the Banach space $\mathbf{B}_{\mu}$, and

$$
G_{0}(t)=\left(L^{-} \varphi\right)(t)-X(t) X_{r}^{-1}\left(t_{0}\right)\left(L^{-} \varphi\right)\left(t_{0}\right)
$$

Definition 3. Operator (13) is called the Green operator of the semihomogeneous $\left(z_{0}=0\right)$ Cauchy problem (4), (5).

Thus, the following theorem is proved:

Theorem 1. Let $L \in G I\left(\mathbf{l}_{\infty}\left(\mathcal{I}, \mathbf{B}_{1}\right), \mathbf{l}_{\infty}\left(\mathcal{I}, \mathbf{B}_{2}\right)\right)$ and let the spaces $\mathbf{l}_{\infty}\left(\mathcal{I}, \mathbf{B}_{1}\right)$ and $\mathbf{l}_{\infty}\left(\mathcal{I}, \mathbf{B}_{2}\right)$ have bases. The Cauchy problem (3), (4) is solvable for those and only those $\varphi(t) \in \mathbf{l}_{\infty}\left(\mathcal{I}, \mathbf{B}_{2}\right)$ that satisfy condition (6) with any $z_{0}=z\left(t_{0}\right), t_{0} \in \mathcal{I}$, and its general solution can be represented in the form

$$
z(t)=X_{0}(t) z_{\mu}+G_{0}(t)+X(t) X_{r}^{-1}\left(t_{0}\right) z_{0}
$$

Remark 1. If the operator equation (2) is everywhere solvable, then $\mathcal{P}_{Y_{L}} \equiv 0$. In this case, the operator $L$ has the bounded right inverse $L_{r}^{-1}$ [5]. If, in addition, the matrix operator $X(t)$ is invertible for any $t \in \mathcal{I}$, then $\mathcal{P}_{N\left(X\left(t_{0}\right)\right)}=0$ and the Cauchy problem (4), (5) is everywhere solvable and has solutions of the form

$$
z(t)=X(t) X^{-1}\left(t_{0}\right) z_{0}+\left(L_{r}^{-1} \varphi\right)(t)-X(t) X^{-1}\left(t_{0}\right)\left(L_{r}^{-1} \varphi\right)\left(t_{0}\right) .
$$


Remark 2. If $L$ is the differential operator $(L z)(t)=z^{\prime}(t)-A(t) z(t)$ acting in the Banach space $C([a ; b], \mathbf{B})$ of functions continuous on $[a ; b]$, then the right inverse $L_{r}^{-1}$ is an integral operator and relation (14) takes the form [3, p. 148]

$$
z(t)=U\left(t, t_{0}\right) z_{0}+\int_{t_{0}}^{t} U(t, \tau) \varphi(\tau) d \tau,
$$

where $U(t, \tau)=U(t) U^{-1}(\tau)$ is the evolution (resolving) operator.

\section{Main Result}

Infinite-Dimensional Normally Solvable Boundary-Value Problems. Consider the linear inhomogeneous boundary-value problem (2), (3).

As shown above, Eq. (2) is solvable under condition (6), and its general solution has the form (9).

Substituting (9) into the boundary condition (3), we obtain the following matrix operator equation for the element $\hat{z}_{0} \in \mathbf{B}_{\mu}$ :

$$
Q \hat{z}_{0}=\alpha-\ell\left(L^{-} \varphi\right)(\cdot),
$$

where $Q=\ell X(\cdot): \mathbf{B}_{\mu} \rightarrow \mathbf{B}$ is the matrix operator obtained by the substitution of the matrix operator $X(t)$ into the boundary condition. The matrix operator $Q$ is bounded as the superposition of the bounded operators $\ell$ and $X(t)$.

Assume that $Q$ is a generalized invertible operator, i.e., $Q \in G I\left(\mathbf{B}_{\mu}, \mathbf{B}\right)$. By virtue of the normal solvability of the operator $Q$, Eq. (14) is solvable for those and only those $\alpha$ and $\varphi(t)$ that satisfy the condition

$$
\mathcal{P}_{Y_{Q}}\left\{\alpha-\ell\left(L^{-} \varphi\right)(\cdot)\right\}=0
$$

where $\mathcal{P}_{Y_{Q}}$ is the projector to the subspace $Y \subset \mathbf{B}$ isomorphic to the null space of the operator $Q^{*}$ adjoint to the operator $Q$. Under condition (16), the operator equation (15) has the solution

$$
\hat{z}_{0}=\mathcal{P}_{N(Q)} z_{\mu}+Q^{-}\left\{\alpha-\ell\left(L^{-} \varphi\right)(\cdot)\right\},
$$

where $z_{\mu}$ is an arbitrary element of the Banach space $\mathbf{B}_{\mu}$ and $Q^{-}$is the generalized inverse of the operator $Q$.

Substituting (17) into (9), we obtain the general solution of the boundary-value problem (2), (3) in the Banach space with basis:

$$
\begin{aligned}
z(t) & =X(t)\left\{\left(\mathcal{P}_{N(Q)} z_{\mu}\right)(t)+Q^{-}\left[\alpha-\ell\left(L^{-} \varphi\right)(\cdot)\right]\right\}+\left(L^{-} \varphi\right)(t) \\
& =X_{\mu}(t) z_{\mu}+(G \varphi)(t)+X(t) Q^{-} \alpha,
\end{aligned}
$$

where $X_{\mu}(t)=X(t) \mathcal{P}_{N(Q)}$ is the resolving operator of the homogeneous $(\varphi(t), \alpha=0)$ boundary-value problem (2), (3).

Definition 4. The operator $(G \varphi)(t)=\left(L^{-} \varphi\right)(t)-X(t) Q^{-} \ell\left(L^{-} \varphi\right)(\cdot)$ is called the generalized Green operator of the semihomogeneous $(\alpha=0)$ boundary-value problem (2), (3). 
Thus, the following statement is true:

Theorem 2. Let $L \in G I\left(\mathbf{l}_{\infty}\left(\mathcal{I}, \mathbf{B}_{1}\right), \mathbf{l}_{\infty}\left(\mathcal{I}, \mathbf{B}_{2}\right)\right)$, let $Q \in G I\left(\mathbf{B}_{\mu}, \mathbf{B}\right)$, and let the Banach spaces $\mathbf{l}_{\infty}\left(\mathcal{I}, \mathbf{B}_{1}\right)$ and $\mathbf{l}_{\infty}\left(\mathcal{I}, \mathbf{B}_{2}\right)$ have bases. Then the homogeneous $(\varphi(t)=0, \alpha=0)$ boundary-value problem (2), (3) has linearly independent solutions of the form

$$
z(t)=X(t) \mathcal{P}_{N(Q)} z_{\mu}=X_{\mu}(t) z_{\mu}
$$

where $X_{\mu}(t)$ is the resolving operator of the homogeneous $(\varphi(t)=0, \alpha=0)$ boundary-value problem and $z_{\mu}$ is an arbitrary element of the Banach space $\mathbf{B}_{\mu}$.

The inhomogeneous boundary-value problem (2), (3) is solvable for those and only those $\varphi(t) \in \mathbf{l}_{\infty}\left(\mathcal{I}, \mathbf{B}_{2}\right)$ and $\alpha \in \mathbf{B}$ that satisfy the conditions

$$
\begin{gathered}
\left(\mathcal{P}_{Y_{L}} \varphi\right)(t)=\Psi(t)(\Phi \varphi)(\cdot)=0, \\
\mathcal{P}_{Y_{Q}}\left\{\alpha-\ell\left(L^{-} \varphi\right)(\cdot)\right\}=0 .
\end{gathered}
$$

The general solution of the problem has the form

$$
z(t)=X_{\mu}(t) z_{\mu}+(G \varphi)(t)+X(t) Q^{-} \alpha,
$$

where $(G \varphi)(t)$ is the generalized Green operator.

Remark 3. In the case where $L z(t)=z^{\prime}(t)-A(t) z(t)$ is an everywhere solvable differential operator, boundary-value problems of the form (2), (3) were considered in $[10,11]$.

Remark 4. Comparing the theorem on the existence of solutions of the Cauchy problem (Theorem 1) and the theorem on the existence of solutions of the boundary-value problem (Theorem 2), we conclude that the Cauchy problem for an operator equation that is not everywhere solvable is a specific boundary-value problem.

The n-and d-Normally Solvable Boundary-Value Problems. Consider two special cases of the boundaryvalue problem (2), (3).

1. Assume that a generalized invertible operator $L$ is $n$-normal $(\operatorname{dim} N(L)=\mu<\infty)$ and acts from the infinite-dimensional Banach space $\mathbf{l}_{\infty}\left(\mathcal{I}, \mathbf{B}_{1}\right)$ into the infinite-dimensional Banach space $\mathbf{l}_{\infty}\left(\mathcal{I}, \mathbf{B}_{2}\right)$. Also assume that a linear bounded vector functional $\ell$ acts from the Banach space $\mathbf{l}_{\infty}\left(\mathcal{I}, \mathbf{B}_{1}\right)$ into the Banach space $\mathbf{B}$. Then the matrix operator $Q$ is also $n$-normal, i.e., the codimension of the kernel $N(Q)$ is finite and the codimension of the kernel $N(Q)^{*}$ of the adjoint matrix operator $Q^{*}$ is infinite.

Theorem 3. Let the operator $L \in G I\left(\mathbf{l}_{\infty}\left(\mathcal{I}, \mathbf{B}_{1}\right), \mathbf{l}_{\infty}\left(\mathcal{I}, \mathbf{B}_{2}\right)\right)$ be $n$-normal, let $Q \in G I\left(\mathbf{B}_{1}, \mathbf{B}\right)$, rank $Q \leq \mu$, and let the Banach spaces $\mathbf{l}_{\infty}\left(\mathcal{I}, \mathbf{B}_{1}\right)$ and $\mathbf{l}_{\infty}\left(\mathcal{I}, \mathbf{B}_{2}\right)$ have bases. Then the homogeneous $(\varphi(t)=0, \alpha=0)$ boundary-value problem (2), (3) has an $r=\mu$-rank $Q$-parameter family of linearly independent solutions of the form

$$
z(t)=X_{r}(t) c_{r}, \quad c_{r} \in R^{r}
$$


where $X_{r}(t)=X(t) \mathcal{P}_{N_{r}(Q)}$ is the resolving operator of the homogeneous $(\varphi(t)=0, \alpha=0)$ boundary-value problem corresponding to problem (2), (3), $\mathcal{P}_{N_{r}}(Q)$ is the operator composed of $r$ linearly independent columns of the matrix projector $\mathcal{P}_{N(Q)}$, and $c_{r}$ is an arbitrary constant vector from the Euclidean space $R^{r}$.

The inhomogeneous boundary-value problem (2), (3) is solvable for those and only those $\varphi(t) \in \mathbf{l}_{\infty}\left(\mathcal{I}, \mathbf{B}_{2}\right)$ and $\alpha \in \mathbf{B}$ that satisfy an infinite number of the linearly independent conditions

$$
\begin{gathered}
\left(\mathcal{P}_{Y_{L}} \varphi\right)(t)=\Psi(t)(\Phi \varphi)(\cdot)=0, \\
\mathcal{P}_{Y_{Q}}\left\{\alpha-\ell\left(L^{-} \varphi\right)(\cdot)\right\}=0 ;
\end{gathered}
$$

in this case, it has an r-parameter family of linearly independent solutions of the form

$$
z(t)=X_{r}(t) c_{r}+(G \varphi)(t)+X(t) Q^{-} \alpha
$$

where $(G \varphi)(t)$ is the generalized Green operator of the semihomogeneous $(\alpha=0)$ boundary-value problem (2), (3).

2. Assume that a generalized invertible operator $L$ acts from the infinite-dimensional Banach space $\mathbf{l}_{\infty}\left(\mathcal{I}, \mathbf{B}_{1}\right)$ into the infinite-dimensional Banach space $\mathbf{l}_{\infty}\left(\mathcal{I}, \mathbf{B}_{2}\right)$, and a linear bounded vector functional $\ell$ acts from the Banach space $\mathbf{l}_{\infty}\left(\mathcal{I}, \mathbf{B}_{1}\right)$ into the Euclidean space $R^{m}$. Then the matrix operator $Q$ is $d$-normal, i.e., the dimension of its kernel $N(Q)$ is finite and the dimension of the kernel $N\left(Q^{*}\right)$ of the adjoint matrix operator $Q^{*}$ is finite and equal to $d \leq m$.

Theorem 4. Let $L \in G I\left(\mathbf{l}_{\infty}\left(\mathcal{I}, \mathbf{B}_{1}\right), \mathbf{l}_{\infty}\left(\mathcal{I}, \mathbf{B}_{2}\right)\right)$, let $Q \in G I\left(\mathbf{B}_{1}, R^{m}\right)$, rank $Q \leq m$, and let the Banach spaces $\mathbf{l}_{\infty}\left(\mathcal{I}, \mathbf{B}_{1}\right)$ and $\mathbf{l}_{\infty}\left(\mathcal{I}, \mathbf{B}_{2}\right)$ have bases. Then the homogeneous $(\varphi(t)=0, \alpha=0)$ boundary-value problem (2), (3) has an infinite family of linearly independent solutions of the form

$$
z(t)=X_{\mu}(t) z_{\mu},
$$

where $X_{\mu}(t)=X(t) \mathcal{P}_{N(Q)}$ is the resolving operator of the homogeneous $(\varphi(t)=0, \alpha=0)$ boundary-value problem corresponding to problem (2), (3) and $z_{\mu}$ is an arbitrary element of the Banach space $\mathbf{B}_{\mu}$.

The inhomogeneous boundary-value problem (2), (3) is solvable for those and only those $\varphi(t) \in \mathbf{l}_{\infty}\left(\mathcal{I}, \mathbf{B}_{2}\right)$ and $\alpha \in \mathbf{B}$ that satisfy an infinite number of the linearly independent conditions

$$
\left(\mathcal{P}_{Y_{L}} \varphi\right)(t)=\Psi(t)(\Phi \varphi)(\cdot)=0
$$

and the $d=m-$ rank $Q$ linearly independent conditions

$$
\mathcal{P}_{Y_{Q_{d}}}\left\{\alpha-\ell\left(L^{-} \varphi\right)(\cdot)\right\}=0
$$

in this case, it has an infinite-dimensional family of linearly independent solutions of the form

$$
z(t)=X_{\mu}(t) z_{\mu}+(G \varphi)(t)+X(t) Q^{-} \alpha,
$$

where $\mathcal{P}_{Y_{Q_{d}}}$ is the $d \times m$ operator matrix composed of the complete system of $d$ linearly independent rows of the matrix $\mathcal{P}_{Y_{Q}}$ and $(G \varphi)(t)$, is the generalized Green operator of the semihomogeneous $(\alpha=0)$ boundary-value problem (2), (3). 
Following [11], we call the boundary-value problems described by Theorems 3 and $4 n$-normally solvable and $d$-normally solvable.

Remark 5. In the case of finite-dimensional boundary-value problems for differential systems of equations in Banach spaces $\left((L z)(t) \equiv z^{\prime}(t)-A(t) z(t)\right)$, problems of this type were considered in [11]. In this case, the generalized inverse operator $L^{-}$admits an integral representation, and the generalized Green operator has the form

$$
(G \varphi)(t)=\int_{a}^{b} K(t, s) \varphi(s) d s-X(t) Q^{-} \ell \int_{a}^{b} K(\cdot, s) \varphi(s) d s
$$

Remark 6. If the functional $\ell$ satisfies the condition [12, p. 15]

$$
\ell \int_{a}^{b} K(\cdot, s) \varphi(s) d s=\int_{a}^{b} \ell K(\cdot, s) \varphi(s) d s
$$

then the generalized Green operator admits the representation

$$
(G \varphi)(t)=\int_{a}^{b} G(t, s) \varphi(s) d s
$$

whose kernel

$$
G(t, s)=K(t, s)-X(t) Q^{-\ell} K(\cdot, s)
$$

is called the generalized Green matrix.

Normally Solvable Boundary-Value Problems for Noetherian Operator Equations. Consider the boundary-value problem (2), (3) under the assumption that $L$ is a linear bounded Noetherian ( $\operatorname{dim} \operatorname{ker} L=\mu<\infty$ and $\left.\operatorname{dim} \operatorname{ker} L^{*}=v<\infty\right)$ operator that acts from the Banach space $\mathbf{l}_{\infty}\left(\mathcal{I}, \mathbf{B}_{1}\right)$ into the Banach space $\mathbf{l}_{\infty}\left(\mathcal{I}, \mathbf{B}_{2}\right)$, $\ell: \mathbf{l}_{\infty}\left(\mathcal{I}, \mathbf{B}_{1}\right) \rightarrow R^{m}$ is a linear bounded vector functional, and $\alpha \in R^{m}$.

Consider the problem of necessary and sufficient conditions for the solvability of the linear inhomogeneous boundary-value problem (2), (3) and the structure of the set of its solutions $z(t) \in \mathbf{l}_{\infty}\left(\mathcal{I}, \mathbf{B}_{1}\right)$.

Since the null spaces $N(L)$ and $N\left(L^{*}\right)$ of the operator $L$ and its adjoint $L^{*}$ are finite-dimensional, they are complementable in the Banach spaces $\mathbf{l}_{\infty}\left(\mathcal{I}, \mathbf{B}_{1}\right)$ and $\mathbf{l}_{\infty}\left(\mathcal{I}, \mathbf{B}_{2}\right)$, respectively, and have finite-dimensional bases. Following [5, pp. 168, 172], we construct the projectors $\mathcal{P}_{N(L)}$ and $\mathcal{P}_{Y_{L}}$.

The Noetherian normally solvable operator equation (2) is solvable for those and only those $\varphi(t) \in \mathbf{l}_{\infty}\left(\mathcal{I}, \mathbf{B}_{2}\right)$ that satisfy the condition

$$
\left(\mathcal{P}_{Y_{L}} \varphi\right)(t)=\Psi(t)(\Phi \varphi)(\cdot)=0
$$

which consists of $v$ linearly independent conditions.

Under condition (19), the general solution of Eq. (2) has the form

$$
z(t)=X_{\mu}(t) c_{\mu}+\left(L^{-} \varphi\right)(t)
$$


where $X_{\mu}(t)$ is the $\infty \times \mu$ operator matrix composed of $\mu$ linearly independent basis vectors of the null space $N(L)$ of the operator $L, \quad c_{\mu} \in R^{\mu}$ is an arbitrary constant vector, and $L^{-}: \mathbf{l}_{\infty}\left(\mathcal{I}, \mathbf{B}_{2}\right) \rightarrow \mathbf{l}_{\infty}\left(\mathcal{I}, \mathbf{B}_{1}\right)$ is the bounded generalized inverse of the Noetherian operator $L$ [8, p. 53].

For solution (20) of the inhomogeneous operator equation (19) to be a solution of the boundary-value problem (2), (3), it is necessary and sufficient that the vector $c_{\mu} \in R^{\mu}$ satisfy the algebraic system

$$
\ell X_{\mu}(\cdot) c_{\mu}+\ell\left(L^{-} \varphi\right)(\cdot)=\alpha
$$

which is obtained by the substitution of solution (20) into the boundary condition (3).

Let $Q=l X(\cdot)$ be an $m \times \mu$ constant matrix, let $\mathcal{P}_{N(Q)}: R^{\mu} \rightarrow N(Q)$ be a $\mu \times \mu$ projector matrix, let $\mathcal{P}_{Q^{*}}: R^{m} \rightarrow N\left(Q^{*}\right)$ be an $m \times m$ projector matrix, and let $Q^{-}$be the $\mu \times m$ generalized inverse of the matrix $Q$.

Using the algebraic equation

$$
Q c_{\mu}=\alpha-l\left(L^{-} \varphi\right)
$$

we determine the constant $c_{\mu} \in R^{\mu}$ for which solution (20) of Eq. (2), which exists under condition (19), is a solution of the boundary-value problem (2), (3). It follows from Theorem 3.9 in [8, p. 92] that Eq. (21) is solvable if and only if

$$
\mathcal{P}_{Y_{d} Q}\left\{\alpha-\ell\left(L^{-}\right)(\cdot)\right\}=0, \quad d=m-\operatorname{rank} Q,
$$

and has an $r$-parameter $(r=\mu-\operatorname{rank} Q)$ family of solutions of the form

$$
c=\mathcal{P}_{N_{r}(Q)} c_{r}+Q^{-}\left\{\alpha-\ell\left(L^{-} \varphi\right)(\cdot)\right\}
$$

where $\mathcal{P}_{Y_{d} Q}$ is the $d \times m$ matrix composed of the complete system of $d$ linearly independent rows of the projector matrix $\mathcal{P}_{Y_{Q}}$, and $\mathcal{P}_{N_{r}}(Q)$ is the $\mu \times r$ matrix composed of the complete system of $r$ linearly independent columns of the projector matrix $\mathcal{P}_{N(Q)}$.

Substituting (22) into (20), we obtain the general solution of the boundary-value problem (2), (3):

$$
z(t)=X_{r}(t) c_{r}+(G \varphi)(t)+X(t) Q^{-} \alpha
$$

Here, $X_{r}(t)=X(t) \mathcal{P}_{N_{r}(Q)}$ is the operator matrix whose columns are the complete system of $r$ linearly independent solutions of the homogeneous $(\varphi(t)=0, \alpha=0)$ boundary-value problem (2), (3), and $G: \mathbf{l}_{\infty}\left(\mathcal{I}, \mathbf{B}_{2}\right) \rightarrow$ $\operatorname{ker} \ell \subset \mathbf{l}_{\infty}\left(\mathcal{I}, \mathbf{B}_{1}\right), \quad(G \varphi)(t)=\left(L^{-} \varphi\right)(t)-X(t) Q^{+} l\left(L^{-} \varphi\right)(\cdot)$, is the generalized Green operator of the semihomogeneous $(\alpha=0)$ boundary-value problem (2), (3).

The following statement is true:

Theorem 5. If rank $Q \leq \min (m, \mu)$, then the homogenous $(\varphi(t)=0, \alpha=0)$ boundary-value problem (2), (3) has $r$ and only $r(r=\mu-\operatorname{rank} Q)$ linearly independent solutions

$$
z(t)=X_{r}(t) c_{r}, \quad c_{r} \in R^{r} .
$$

The inhomogeneous boundary-value problem (2), (3) with Noetherian operator $L: \mathbf{l}_{\infty}\left(\mathcal{I}, \mathbf{B}_{1}\right) \rightarrow \mathbf{l}_{\infty}\left(\mathcal{I}, \mathbf{B}_{2}\right)$ is solvable for those and only those $\varphi(t) \in \mathbf{l}_{\infty}\left(\mathcal{I}, \mathbf{B}_{2}\right)$ and $\alpha \in R^{m}$ that satisfy the $v+d$ linearly independent conditions 


$$
\begin{gathered}
\left(\mathcal{P}_{Y_{L}} \varphi\right)(t)=0, \\
\mathcal{P}_{Y_{d} Q}\left\{\alpha-\ell\left(L^{-} \varphi\right)(\cdot)\right\}=0, \quad d=m-\operatorname{rank} Q ;
\end{gathered}
$$

in this case, it has the r-parameter family of linearly independent solutions

$$
z(t)=X_{r}(t) c_{r}+(G \varphi)(t)+X(t) Q^{+} \alpha
$$

Now consider the boundary-value problem (2), (3) under the assumption that the operator equation (2) is everywhere solvable. This implies that $R(L)=\mathbf{l}_{\infty}\left(\mathcal{I}, \mathbf{B}_{2}\right)$, and, hence, $\mathcal{P}_{Y_{L}} \equiv 0$. According to Corollary 2 of Theorem 2.3 in [8, p. 55], the operator equation $(L z)(t)=\varphi(t)$ is solvable for any $\varphi(t) \in \mathbf{l}_{\infty}\left(\mathcal{I}, \mathbf{B}_{2}\right)$ and has the solution

$$
z(t)=X_{\mu}(t) c_{\mu}+\left(L_{r}^{-1} \varphi\right)(t), \quad c_{\mu} \in R^{\mu},
$$

where $L_{r}^{-1}$ is the linear bounded right inverse operator.

Since the equation $(L z)(t)=\varphi(t)$ is everywhere solvable, Theorem 5 is simplified and can be reformulated as follows:

Theorem 6. If $\operatorname{rank} Q \leq \min (m, \mu)$, then the homogeneous $(\varphi(t)=0, \alpha=0)$ boundary-value problem (2), (3) has $r$ and only $r(r=\mu-\operatorname{rank} Q)$ linearly independent solutions

$$
z(t)=X_{r}(t) c_{r}, \quad c_{r} \in R^{r}
$$

The inhomogeneous boundary-value problem (2), (3) for the everywhere solvable operator equation $L z=\varphi$ is solvable for those and only those $\varphi(t) \in \mathbf{l}_{\infty}\left(\mathcal{I}, \mathbf{B}_{2}\right)$ and $\alpha \in R^{m}$ that satisfy the $d$ linearly independent conditions

$$
\mathcal{P}_{Y_{Q_{d}}}\left\{\alpha-\ell\left(L_{r}^{-1} \varphi\right)(\cdot)\right\}=0, \quad d=m-\operatorname{rank} Q
$$

in this case, it has the r-parameter family of solutions

$$
z(t)=X_{r}(t) c_{r}+(G \varphi)(t)+X(t) Q^{-} \alpha
$$

where $(G \varphi)(t)=\left(L_{r}^{-1} \varphi\right)(t)-X(t) Q^{-} \ell\left(L_{r}^{-1} \varphi\right)(\cdot)$ is the generalized Green operator.

Remark 7. If $\mu=m$ and $\operatorname{rank} Q=\mu$, then $\operatorname{det} Q \neq 0\left(P_{Y_{Q}} \equiv 0\right.$ and $\left.P_{N(Q)} \equiv 0\right)$ and $Q^{-}=Q^{-1}$. In this case, the boundary-value problem (2), (3) is not only everywhere but also uniquely solvable, and the generalized Green operator turns into the Green operator

$$
(G \varphi)(t)=\left(L_{r}^{-1} \varphi\right)(t)-X(t) Q^{-1} \ell\left(L_{r}^{-1} \varphi\right)(\cdot)
$$

\section{REFERENCES}

1. I. Ts. Gokhberg and N. Ya. Krupnik, Introduction to the Theory of One-Dimensional Singular Integral Operators [in Russian], Shtiintsa, Kishinev (1973).

2. M. I. Kadets and B. S. Mityagin, "Complementable subspaces in Banach spaces," Usp. Mat. Nauk, 28, Issue 6, 77-94 (1973). 
3. Yu. M. Daletskii and M. G. Krein, Stability of Solutions of Differential Equations in a Banach Space [in Russian], Nauka, Moscow (1970).

4. M. M. Grinblyum, "Biorthogonal systems in a Banach space," Dokl. Akad. Nauk SSSR, 47, No. 2, 79-82 (1945).

5. V. F. Zhuravlev, "Solvability criterion and representation of solutions of $n$-normal and $d$-normal linear operator equations in a Banach space," Ukr. Mat. Zh., 62, No. 2, 167-182 (2010); English translation: Ukr. Math. J., 62, No. 2, 186-202 (2010).

6. V. F. Zhuravlev, "Solution of normally solvable operator equations in Banach spaces with basis," Dokl. Ros. Akad. Nauk, 352, No. 3, 304-306 (1997).

7. A. A. Boichuk and A. M. Samoilenko, Generalized Inverse Operators and Fredholm Boundary-Value Problems, VSP, Utrecht (2004).

8. A. A. Boichuk, V. F. Zhuravlev, and A. M. Samoilenko, Generalized Inverse Operators and Noetherian Boundary-Value Problems [in Russian], Institute of Mathematics, Ukrainian National Academy of Sciences, Kiev (1995).

9. L. A. Lyusternik and V. I. Sobolev, A Brief Course in Functional Analysis [in Russian], Vysshaya Shkola, Moscow (1982).

10. V. F. Zhuravlev, "Linear boundary-value problems for differential equations in Banach spaces," in: Abstracts of the International Scientific Conference "Differential Equations, Theory of Functions, and Their Applications" Dedicated to the 70th birthday of Academician A. M. Samoilenko (June 18-21, 2008), Melitopol' (2008), p. 50.

11. O. A. Boichuk and E. V. Panasenko, "Boundary-value problems for differential equations in a Banach space," Nelin. Kolyvannya, 12, No. 1, 16-19 (2009); English translation: Nonlin. Oscillations, 12, No. 1, 15-18 (2009).

12. V. S. Korolyuk and A. F. Turbin, Mathematical Foundations of Phase Lumping of Complex Systems [in Russian], Naukova Dumka, Kiev (1978). 